\title{
Associations of COVID-19 Pandemic with Clinical Manifestations among the Uninfected Pregnant Women in China: A Combined Cohort Study
}

Bin Wang ${ }^{1}$, Hang An², Huanqing $\mathrm{Hu}^{3}$, Wei Zhao ${ }^{3}$, Bahabaike Jiangtulu ${ }^{1}$, Shuo Wang ${ }^{4}$, Jiamei Wang ${ }^{4}$, Junxi Chen ${ }^{5}$, Manman Long ${ }^{5}$, Zewu Li ${ }^{5}$, Yu Jin $^{5}$, Yuhuan Li $^{5}$, Huiting $\mathrm{Chen}^{5}$, Tao Xue ${ }^{6}$, Xiqing $\mathrm{Li}^{7}$, Kexin $\mathrm{Li}^{8}$, Wei $\mathrm{Du}^{9}$, Suhong $\mathrm{Gao}^{4}$, Jiangli $\mathrm{Di}^{10}$, Xiaohong $\mathrm{Liu}^{4}$, Rongwei $\mathrm{Ye}^{11}$, and Zhiwen $\mathrm{Li}^{2}$

${ }^{1}$ Institute of Reproductive and Child Health, Peking University/ Key Laboratory of Reproductive Health, National Health Commission of the People's Republic of China, Beijing 100191, P.R. China

${ }^{2}$ Institute of Reproductive and Child Health/Ministry of Health Key Laboratory of Reproductive Health, Peking University Health Science Center

${ }^{3}$ Chinese Center for Disease Control and Prevention

${ }^{4}$ Affiliation not available

${ }^{5}$ Institute of Reproductive and Child Health, Peking University/ Key Laboratory of

Reproductive Health, Peking University

${ }^{6}$ Peking University Health Science Centre

${ }^{7}$ College of Urban and Environmental Sciences, Peking University

${ }^{8}$ Institute of Geographic Sciences and Natural Resources Research CAS

${ }^{9}$ Key Laboratory of Geographic Information Science of the Ministry of Education, School of Geographic Sciences, East China Normal University

${ }^{10}$ National center for women and children's health, China CDC

${ }^{11}$ Institute of Reproductive and Child Health, Peking University Health Science Center

October 16, 2020

\begin{abstract}
Objective: To investigate whether the COVID-19 pandemic would significantly affect pregnancy-associated factors in uninfected pregnant women in China. Design: A combined cohort study Setting: Six sites in China Population: Uninfected pregnant women participating in the programs with a singleton birth and delivery or termination from January 1 to May 312019 (pre-pandemic period, P-2019) and from January 1 to May 312020 (pandemic period, P-2020) (n = 32,277). Methods: The associations of pregnancy-associated outcomes and the COVID-19 were assessed by poisson regression, linear regression or log-binomial regression. Main Outcomes Measures: The number of prenatal examinations (NPE), delivery gestational week (DGW), caesarean section (CS), preterm birth, macrosomia. Results: For Hubei, the NPE and DGW were negatively associated with the COVID-19 pandemic, whereas the CS and preterm birth rates were positively associated with the COVID-19, with adjusted relative risks (aRRs) of 1.11 [95\% confidence interval (CI) 1.06-1.17] and 1.37 (95\% CI: 1.02-1.84) respectively. For Guangdong, the associations of CS and preterm birth with the COVID-19 were similar in Hubei. Limited associations were evident in other areas while a positive association with macrosomia was observed in Beijing [aRR = 1.26 (95\% CI: 1.03-1.55)]. Conclusions: The CS and preterm birth rates increased slightly in areas that were more affected by the pandemic than other
\end{abstract}


areas. NPEs were not significantly interrupted and most maternal and neonatal clinical characteristics were within the normal ranges. Appropriate interventions should be considered to protect pregnant women. Keywords: COVID-19, pregnancy outcome, neonatal outcome, uninfected pregnant women

\section{Hosted file}

Main text.pdf available at https://authorea.com/users/367729/articles/487060-associationsof-covid-19-pandemic-with-clinical-manifestations-among-the-uninfected-pregnant-womenin-china-a-combined-cohort-study 